\title{
TEMPERATUREN DES BEGEHRENS. SINNLICHE PRÄSENZ UND KULTURELLE REPRÄSENTATIONEN
}

\section{ABSTRACT. Temperatures of desire. Sensual presence and cultural representations}

The aim of the article is to present the interdisciplinary theoretical framework for the analysis of the constellation of temperature and desire as well as its representations in literature and culture, which have not been the subject of a broad reflection in the area of literary and cultural studies so far. The question therefore is: What is the role of the literary, iconographic and media discourses of temperature in the processes of the creating and transformations of emotional cultures, social phenomena, political conditions, and last but not least in the critical reflection of these processes.

KEYWORDS: temperature, desire, love, gender, society, literature, culture, media

„Das Thermometer beschäftiget jedermann [...].“”

(J.W. Goethe)

Ein Seufzer lief Schlittschuh auf nächtlichem Eis

und träumte von Liebe und Freude.

Es war an dem Stadtwall, und schneeweiß

glänzten die Stadtwallgebäude.

Der Seufzer dacht' an ein Maidelein

und blieb erglühend stehen.

Da schmolz die Eisbahn unter ihm ein -

und er sank - und ward nimmer gesehen. ${ }^{2}$

\footnotetext{
${ }^{1}$ Johann Wolfgang Goethe: Versuch einer Witterungslehre. In: Goethes Werke: Vollständige Ausgabe letzter Hand. Bd. 51. Stuttgart/Tübingen 1834, S. 247-282, hier S. 274.
} 
Das Gedicht Der Seufzer von Christian Morgenstern den folgenden Erwägungen voranzustellen, scheint uns aus zweifachem Grund berechtigt zu sein. Zum einen, weil hier zwei Vorstellungen bzw. Begriffe poetisch zusammengebracht werden, die offensichtlich seit jeher zusammengehören, die jedoch bis dahin in dieser Konfiguration selten zum Gegenstand einer Analyse wurden - Begehren und Temperatur. Zum anderen, weil Morgenstern gelingt, in der knappen Form die ganze Temperaturskala des Begehrens auf den Punkt zu bringen - das Schwanken zwischen einer Imagination des Erglühens und der (erfahrenen) klirrenden Kälte.

Wie das Beispiel zeigt, erweckt die Konstellation der Begriffe Begehren und Temperatur ganz bestimmte Assoziationen, die in unserer Gefühlskultur eng mit Liebe und Erotik verbunden sind und die eine bemerkenswert lange Tradition haben. Heiße Liebe, entflammte Leidenschaften, glühende Erotik, brennendes Verlangen in diesen Kategorien versucht man immer wieder dem Begehren und seinen Realisierungsformen - Liebe und Sexualität - beizukommen, sie zu beschreiben, zu kommunizieren oder zu imaginieren. Doch werden die Erhitzungsszenarios gelegentlich durch „abkühlende“ Diskurse begleitet, die das Ziel verfolgen, eine dem Begehren gegenüber feindliche Atmosphäre zu erzeugen bzw. ein Liebesfeuer zu löschen, sei es mit Hilfe einer „Kälte-Pädagogik ${ }^{\star 3}$, sei es mit Hilfe von anderen moralisierenden, juristischen oder medizinischen - Diskursen. Es sei zu betonen, dass Wärme und Kälte als Grundphänomene der menschlichen Erfahrung die Kategorien sind, die sowohl in ihrer sinnlichen Präsenz als auch auf der Ebene der diskursiven Praxis und der künstlerischen Repräsentationen das Begehren immer wieder begleitet und geformt haben. Die messbaren, realen Temperaturwerte der Luft oder des Körpers, die eine Grundlage für sinnliche Eindrücke und Erlebnisse, Affekte und Emotionen, Einschätzungen und Diagnosen, schließlich für Einschränkungen und Kontrollmechanismen schaffen, bilden ein relevantes Motiv in zahlreichen kulturellen Texten, in denen sich Begehren manifestiert - verstanden im Sinne eines umfassenden sozialen Phänomens, das über intime Zusammenhänge hinausgeht und auch politisch-ideologische Machtdimensionen einschließt.

Die im Hinblick auf Temperaturen des Begehrens evozierten und künstlerisch inszenierten assoziativen Gedankenketten von den Intensitäten des Verlangens und Fühlens - von Wärme und Hitze der Leidenschaft bzw. von Kälte und Coolness der emotionalen Indifferenz oder der Affektkontrolle ${ }^{4}$ können einerseits in der

\footnotetext{
${ }^{2}$ Christian Morgenstern: Der Seufzer. In: Rainer Brambach (Hrsg.): Moderne deutsche Liebesgedichte. Von Stefan George bis zur Gegenwart. Zürich 1980, S. 17.

${ }^{3}$ Jos van Ussel: Sexualunterdrückung. Geschichte der Sexualfeindschaft. Gießen 1977, S. 153.

${ }^{4}$ Zur Karriere der Coolness vgl. Ulla Haselstein: Coolness. In: Gunter Gebauer, Markus Edler (Hrsg.): Sprachen der Emotionen. Kultur, Kunst, Gesellschaft. Frankfurt a. M./New York 2014, S. 211-225. Haselstein verweist darauf, dass Kälte schon in den antiken literarischen Texten als Metapher genutzt wurde, um den Verstand den „heißen“ Affekten gegenüberzustellen. In der Emotionsforschung vollzieht
} 
ontogenetischen Dimension in Bezug auf das Individuum untersucht werden. Abgesehen von der metaphorischen Konzeptualisierung der Gefühlskulturen bzw. Darstellungen von fiebernden Verliebten ist es andererseits möglich, über das Begehren im Zusammenhang mit meteorologischen, klimatisch-jahreszeitlichen, landschaftlichen, topographisch-räumlichen und nicht zuletzt mit soziokulturellen Bedingungen nachzudenken. Temperaturen bezeichnen folgerichtig zum einen wirkliche Wärme- und Kälte-Grade im meteorologischen oder technischen Sinn, zum anderen aber emotionale Phänomene mit dem ihnen zugrunde liegenden gendering - einem Prinzip, nach dem Wärme und Kälte als entsprechend weiblich und männlich konnotiert sind.

Durch die Dimension der Erfahrung und der sinnlichen Wahrnehmung, die über objektive Messbarkeit hinausgeht, rücken die Temperaturen in die Nähe des Atmosphären-Konzepts, wie es in der Philosophie und in den Kulturwissenschaften seit einigen Jahrzehnten entwickelt, diskutiert und mit Raum ${ }^{5}$, Wetter, Körper/Leib, Gefühl, etc. in Verbindung gebracht wird. Gernot Böhme definiert die Atmosphäre als „die Sphäre gespürter leiblicher Anwesenheit" ${ }^{\text {"6 }}$ und macht darauf aufmerksam, dass das Wetter immer etwas Regionales und Singuläres, eine subjektive Tatsache darstellt, obwohl man für dessen Erscheinen objektive Bedingungen angeben kann. ${ }^{7}$ Eine Untersuchung von historischen und gegenwärtigen kulturellen Texten, die sich dem so profilierten meteorologisch-kulturwissenschaftlichen Konzept von Liebe und Begehren verschriebe, müsste sich an der Schnittstelle zwischen Gender-, Medien-, Raum- und Emotionsforschung positionieren und könnte darüber hinaus auf Denktraditionen der Psychoanalyse ${ }^{8}$ sowie auf philosophische Theorien des Begehrens zurückzugreifen. ${ }^{9}$

sich allerdings eine Auflösung der Dichotomie von Gefühl und Ratio, die immer auch geschlechtsspezifisch aufgeladen wurde - vgl. Katharina Scherke: Auflösung der Dichotomie von Rationalität und Emotionalität? Wissenschaftssoziologische Anmerkungen. In: Sabine Flick, Annabelle Hornung (Hrsg.): Emotionen in Geschlechterverhältnissen. Affektregulierung und Gefühlsinszenierung im historischen Wandel. Bielefeld 2009, S. 23-42.

${ }^{5}$ Vgl. Burkhard Meyer-Sickendiek: Gefühlstiefen: Aktuelle Perspektiven einer vergessenen Dimension der Emotionsforschung. In: Gertrud Lehnert (Hrsg.): Raum und Gefühl. Der Spatial Turn und die neue Emotionsforschung. Bielefeld 2011, S. 26-48.

${ }^{6}$ Gernot Böhme: Das Wetter und die Gefühle. Für eine Phänomenologie des Wetters. In: Kerstin Andermann, Undine Eberlein (Hrsg.): Gefühle als Atmosphären. Neue Phänomenologie und philosophische Emotionstheorie. Berlin 2011, S. 153-166, hier S. 155.

${ }^{7}$ Ebd., S. 154.

${ }^{8}$ Der Begriff des Begehrens steht insbesondere im Zentrum des Ansatzes von Lacan. Vgl. z. B. Peter Widmer: Subversion des Begehens. Eine Einführung in Jacques Lacans Werk. Berlin 1997.

${ }^{9}$ Vgl. beispielsweise Roger Scruton: Sexual desire. A moral philosophy of the erotic, New York 1986. Scruton rekapituliert abendländische Auffassungen des Begehrens, um seine These zu begründen, der zufolge es nicht nur intentional auf die verkörperte Person des anderen gerichtet sei, sondern auch die interpersonale Begegnung mit dem seinerseits einen selbst begehrenden Anderen suche. 
Die letzteren bieten ein breites Spektrum von Ansätzen, besonders im Rahmen der neulich in der französischen Philosophie formulierten ${ }^{10}$ „Phänomenologien des Begehrens" “11 , sowie der leibphänomenologisch fundierten atmosphärischen Theorie der Gefühle, die von Hermann Schmitz entwickelt ${ }^{12}$ und von vielen Forscher/innen kritisch diskutiert, ergänzt oder modifiziert wird. ${ }^{13}$ Die „neue Phänomenologie“ versteht sich nicht mehr als Deskription universalisierbarer Strukturen des Bewusstseins, sondern berücksichtigt kulturelle und soziale Faktoren, die eine singuläre Situation ausmachen - sie ist angesiedelt zwischen Diskurs und Erfahrung und meint dabei die „Erfahrung eines situierten, leiblichen, geschlechtlichen Individuums ". ${ }^{14}$ Gerade in der phänomenologischen Argumentation wird gern auf diverse Metaphern aus dem sensorischen oder meteorologischen Bereich rekurriert ${ }^{15}-$ beispielsweise spricht José Ortega y Gasset in seinen Meditationen über die Liebe von

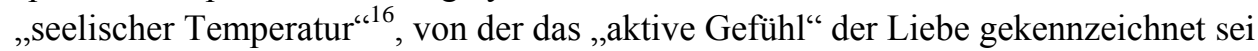
und von einer ,günstigen Atmosphäre“"17, durch die das Objekt der Liebe gehüllt werde. Doch unabhängig von methodologischen Grundlagen kann wohl in Bezug auf diverse historische und aktuelle designs of desire mit Martha Nussbaum behauptet werden: „Kulturelle Gebilde berühren nicht nur die theoretische Erklärung des Begehrens, sondern auch die Erfahrung des Begehrens selbst sowie die Art und Weise, in der man sich selbst als begehrenden Akteur erlebt."“18

\footnotetext{
${ }^{10}$ Vgl. Hans-Dieter Gondek, Lászlo Tengelyi (Hrsg.): Neue Phänomenologie in Frankreich. Berlin 2011.

${ }^{11}$ Esther Hutfless: In Dir mehr als Dich. Phänomenologien des Begehrens zwischen diskursiver Produktion und leiblichem Zur-Welt-Sein. Würzburg 2011.

${ }^{12}$ Vgl. beispielsweise Hermann Schmitz: Gefühle als Atmosphären und das affektive Betroffensein von innen. In: Hinrich Fink-Eitel, Georg Lohmann (Hrsg.): Zur Philosophie der Gefühle. Frankfurt a. M. 1993, S. 33-56. Nach Schmitz sind Gefühle als „ortlos ergossene Atmosphären zu bestimmen, die einen Leib, den sie einbetten, in der Weise des affektiven Betroffenseins heimsuchen, wobei dieses die Gestalt der Ergriffenheit annimmt" (Hermann Schmitz: Der Gefühlsraum. System der Philosophie III/2. Bonn 1969, S. 343).

${ }^{13}$ Christoph Demmerling schlägt beispielsweise vor, Gefühle - ebenso wie Schmitz - als subjektive Zustände, aber - polemisch gegen Schmitz - auch nicht als objektive Phänomene aufzufassen. Nach ihm haben Gefühle intersubjektiven Charakter, was mit der Idee, sie als Atmosphären betrachten zu können, durchaus vereinbar ist. (Vgl. Christoph Demmerling: Gefühle, Sprache und Intersubjektivität. Überlegungen zum Atmosphärenbegriff der Neuen Phänomenologie. In: Andermann, Eberlein: Gefühle als Atmosphären, S. 43-55).

${ }^{14}$ Hutfless: In Dir mehr als Dich, S. 13.

${ }^{15} \mathrm{Zu}$ den phänomenologischen Metaphern der Liebe vgl. Íngrid Vendrell Ferran: Metaphern der Liebe. Alexander Pfänder und Hermann Schmitz. In: Andermann, Eberlein: Gefühle als Atmosphären, S. 245-264.

${ }^{16}$ José Ortega y Gasset: Über die Liebe. Meditationen. Deutsch von Helene Weyl. Stuttgart 1957, S. 102.

${ }^{17}$ Ebd., S. 104.

${ }^{18}$ Martha C. Nussbaum: Konstruktion der Liebe, des Begehrens und der Fürsorge. Drei philosophische Aufsätze. Übers. Joachim Schulte. Stuttgart 2002, S. 169.
} 
Es ist nicht allein Martha Nussbaum, die im Kontext der Reflexionen über Liebe und Begehren auf die Angewiesenheit der Philosophie auf Literatur aufmerksam macht. ,Wir lernen nicht nur aus Literatur, sondern wir erfahren Literatur auch, und durch ihre Brille die Welt, um ihrer selbst willen“19, schreibt Angelika Krebs, die an Nussbaums literaturästhetischen Ansatz anknüpft, um „Liebe als dialogisches Teilen des Lebens genauer zu denken “20. Sie verfolgt Transformationen der Liebe und des Begehrens in Kultur und Literatur - Dynamiken, die sich übrigens auch oft innerhalb der Wärme- und Kälte-Metaphorik diskutieren lassen. Eine „Entwertung oder Umwertung althergebrachter gesellschaftlicher Kodizes im Umgang mit Liebe und Sexualität scheint geradezu zu den Insignien der Moderne zu gehören“, konstatiert Thomas Homscheid. ${ }^{21}$ Diese Entwicklungslinie führt zur gegenwärtigen Lage der Liebeskultur, die durch eine überbordende Präsenz des Sexuellen gekennzeichnet ist, was nicht unbedingt mit der Erfüllung des Begehrens nach dem Anderen einhergeht - eine Situation, die das Englische auf die treffende Formel oversexed and underfucked $^{22}$ bringt, und die in zahlreiche Texte der Gegenwartsliteratur Eingang gefunden hat. Jean Baudrillard hat die Tendenz zur allumfassenden Sexualisierung bereits in den 1970er Jahren festgehalten, als er schrieb: „Alles wird sexualisiert und findet darin gewissermaßen ein Terrain für Spiel und Abenteuer. Überall spricht 'Es' und alle Diskurse sind gleichsam ewige Sex- und Begehrenskommentare. ${ }^{23}$ Der Philosoph sieht gleichsam prophetisch eine „Ordnung des Ludischen“ am Werke und definiert das Ludische als „Ort einer kalten Verführung“: Es sei „der 'narzisstische' Zauber der Elektronik- und Informationssysteme, der kalte Zauber des Mediums und des Terminals, denn Terminals sind wir alle, isoliert in der manipulatorischen Selbstverführung aller Schalthebel, die uns umgeben“. ${ }^{24}$

In der Gegenwartsliteratur, die im Kontext der Transformation von tradierten Begehrensmustern angesiedelt ist, wird das Begehren mehrfach nicht realisiert die Partner/innen leben eher nebeneinander als miteinander, weder schlafen sie miteinander noch wollen berührt werden. Terésia Moras Alle Tage (2004), Eva Menasses Lässliche Todsünden (2009), Reinhard Jirgls Die Stille (2009) könnten - neben zahlreichen anderen literarischen Werken - als Beispiele hierfür herangezo-

${ }^{19}$ Angelika Krebs: Zwischen Ich und Du. Eine dialogische Philosophie der Liebe. Berlin 2015, S. 234 [Kursive im Original].

${ }^{20}$ Ebd., S. 12.

${ }^{21}$ Thomas Homscheid: Sexualzynismen in der Literatur des 20. Jahrhunderts. Am Beispiel von Erich Kästners Fabian (1931). In: Stefan Neuhaus (Hrsg.): Figurationen der Liebe in Geschichte und Gegenwart, Kultur und Gesellschaft. Würzburg 2012, S. 257-272, hier S. 261.

${ }^{22}$ Vgl. Iris Osswald-Rinner: Oversexed und underfucked. Über die gesellschaftliche Konstruktion der Lust. Wiesbaden 2011.

${ }^{23}$ Jean Baudrillard: Von der Verführung. Aus dem Französischen von Michaela Meßner. Berlin 2012, S. 207.

${ }^{24}$ Ebd., S. 188 [Kursive im Original]. 
gen werden. Im Roman Liebeserklärung (2003) von Michael Lentz ist nicht nur von einem ,jahrelangen Ehezustand der absoluten Ficklosigkeit“ ${ }^{\star 25}$ die Rede, sondern es wird auch eine Diagnose über „unsere so genannte westliche Bumskultur“ formuliert - sie sei nämlich „ziemlich vorn Hund, ziemlich kaputt ${ }^{\text {‘26 }}$. Für die Reflexionen über Intimität bietet Deutschland in diesem Liebes- und Krisenroman eine ,erkaltete Kulisse $^{\text {‘27 }}$ - „es ist traurig kalt, es steht uns ein fickloser Winter bevor ${ }^{\text {“28 }}$, lautet das Urteil. Das Bild wird vielfach variiert - in den Zügen fällt etwa die Heizung aus, der Erzähler attestiert der geliebten Frau eine „Nichtwärme“ ${ }^{\text {“29 }}$, sie findet ihn wiederum „eiskalt ${ }^{\star 30}$. Das Begehren wird hier dargestellt, als wäre es von vornherein im Vergehen begriffen, als wäre die oftmals angesprochene Kälte unauslöschbar in das Script eingeschrieben. Zugleich ist der kompromisslose Bericht über das Scheitern einer Liebe - paradoxerweise - die Suche nach einer Sprache der Liebe, die auffallend oft auf meteorologische oder räumliche Konstellationen zurückgreift: „Nordpol Südpol sind wir, nie waren wir weiter weg, nie kälter, eine an Erlösung grenzende Sicherheit, auseinander zu gehen, nicht mehr zueinander zu finden“. 31

Mit dem bedeutungsschweren Titel des Romans Feuer brennt nicht (2009) nimmt Ralf Rothmann eine Diagnose über zeitspezifische Befindlichkeit moderner Geliebter vorweg. „»Feuer brennt nicht! Feuer flackert oder leuchtet oder raucht. Brennen tut was anderes...« «32 - macht dem schriftstellernden, hypochondrischen Protagonisten seine Lebensgefährtin zum Vorwurf und meint zum einen ihre brennende Wunde - die von dem Mann verweigerte Verbindlichkeit der Beziehung, zum anderen dessen brennenden Wunsch nach einem pluralistischen Liebes- und Begehrenskonzept bar aller „Chemie des Betrugs“333. Die von den Figuren perfektionierte Fähigkeit, „[s]ich ein Fieber einzureden, bis das Quecksilber im Thermometer steigt $^{\text {‘34 }}$, hat außer der physischen auch eine symbolische Dimension - das (vergebliche) Jagen nach Erfahrungen, die ,an den Glanz und das Feuer [d]er Vorstellungen“35 herankämen. So endet Rothmanns dunkle „Chronik des erotischen Begehrens ${ }^{\text {‘36 }}$ mit einem düsteren Kälte-Szenario, ähnlich wie die Liebesgeschichte in Peter Stamms Agnes (1998). Agnes, über die der in sie verliebte namenlose Erzähler eine

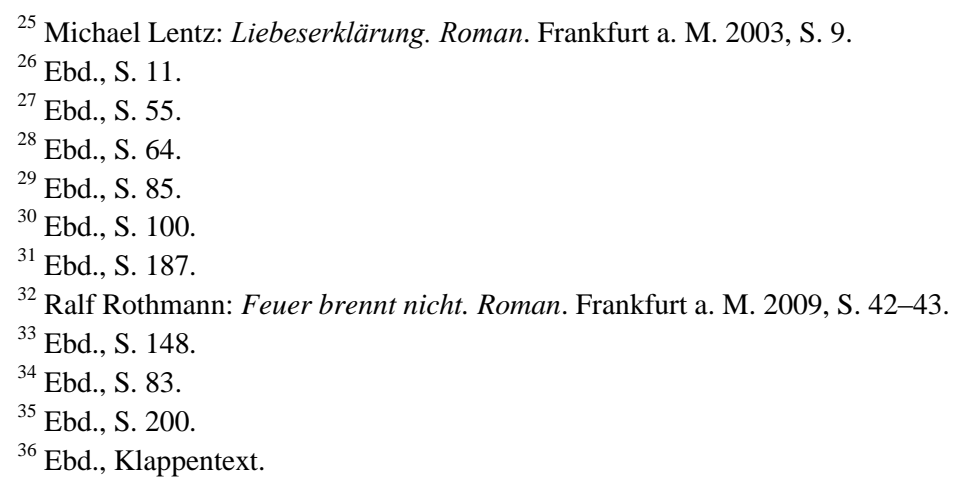


Geschichte schreibt, ist tot. Es wird erzählt, wie sie in der Winterlandschaft herumirrt und nachts im Wald eine Stelle findet, wo sie sich hinlegt und das Gesicht in den Schnee drückt:

Langsam gewann sie das Gefühl zurück, erst in den Füßen, in den Händen, dann in den Beinen und Armen, es breitete sich aus, wanderte durch ihre Schultern und ihren Unterleib zu ihrem Herzen, bis es ihren ganzen Körper durchdrang und es ihr schien, als liege sie glühend im Schnee, als müsse der Schnee unter ihr schmelzen. ${ }^{37}$

Die polnische alternative Liedermacherin Maria Peszek singt in einem Lied aus ihrer umstrittenen, notabene heiß diskutierten Platte Maria Awaria (2008) von der ,arktischen Erektion" eines Eisbären, die das Liebesobjekt nicht etwa zum Glühen, sondern ,unter Eis“ bringt. ${ }^{38}$ Doch bereits in Judith Hermanns Sommerhaus, später (1998) begegnen sich die Paare immer wieder nicht nur im Herbst und Winter in eiskalten Räumen, sondern auch mit Kälte, Stummheit und Emotionslosigkeit. Sie schauen sich an ,ohne Erotik, ohne Flirt, ohne Schmelz ${ }^{639}$ und in der Erinnerung an das gelebte Begehren heißt es: „Vielleicht waren diese Nächte auch einfach nur kalt und in zynischer Weise unterhaltsam. Heute aber kommen sie mir so wichtig vor und so verloren, daß es mich schmerzt. ${ }^{\text {“40 }}$ So sieht sich Volker Demuth in seiner Besprechung des Erzählbandes zur Spekulation über eine Zäsur provoziert, die durch einen kühl-distanzierten Liebeshabitus und durch Berührungslosigkeit gekennzeichnet ist: „Und nun, ein Um-Erzählen von Liebe zur Liebeslosigkeit, von brennender Sehnsucht zur Gefühlsvereisung?“41

Die Frage ist insofern bedeutsam, als die Gegenwartsliteratur das Begehren einerseits tatsächlich als prekär darstellt, andererseits diesem Umstand auch auf den Grund zu gehen versucht. Häufig wird eine kalkulierte Nähe thematisiert, die permanent Gegenstand von Reflexionen und Gesprächen ist, und immer wieder neu bewertet oder „optimalisiert“ wird. Dem Befund über eine krisenhafte Beziehung aus Lentz' Liebeserklärung könnte man daher eine allgemeinere Bedeutung attestieren: „Die Beziehung ist nicht nur zu einem bloßen Gespräch über diese so genannte Beziehung geworden, diese so genannte Beziehung ist zu einem Bilanzieren, zu einer Schlussstrichaddition dieser so genannten Beziehung geworden. “42 Das reflexiv-analytische Moment wird mitunter durch ein ,alternatives“ Begehren ergänzt, das in Evelyn Schlags Roman Das L in Laura (2003) folgendermaßen charakterisiert wird:

\footnotetext{
${ }^{37}$ Peter Stamm: Agnes. Roman. Frankfurt a. M. 2015, S. 152 [Kursive im Original].

${ }^{38}$ Maria Peszek: Maria Awaria (2008), der Song Miś [Bär].

${ }^{39}$ Judith Hermann: Sommerhaus, später. Erzählungen. Frankfurt a. M. 2009, S. 57.

${ }^{40}$ Ebd., S. 70.

${ }^{41}$ Volker Demuth: Unnahbare Nähe. Von Liebe und der neuen Intimitätsökonomie. In: allmende, Zeitschrift für Literatur 86 (2010), S. 39-47, hier S. 40.

${ }^{42}$ Lentz: Liebeserklärung, S. 99.
} 
Eine Liebesbeziehung ohne körperliche Liebe, ohne sexuelles Begehren, nur mit dem Begehren, soviel über die andere Person zu erfahren wie möglich und an ihrem Leben teilzunehmen - what are you having for dinner tonight? $?^{43}$

Auch dieser Gedanke, der sich auf erotische Begegnungen im Cyberspace bezieht, scheint zumindest in den gegenwärtigen Inszenierungen von Liebe und Begehren eine breitere Bedeutung zu haben und auch herkömmlichen Offline-Beziehungen gerecht zu werden.

Die Rolle der literarischen, ikonographischen und medialen Temperatur-Diskurse im Prozess der Transformationen von Gefühlskulturen und Genderverhältnissen mag zwar auf den ersten Blick einleuchten, stellt aber ein kulturwissenschaftliches Forschungsdesiderat dar. Es lässt sich die These aufstellen, dass das Begehren, dessen Wahrnehmung, Artikulations- und Kommunikationsweisen in der Epoche der Klimaerwärmung kaum noch mit Glut und Feuer, sondern vielmehr mit Kälte, Distanz und Ironie verbunden sind. Wohl in diesem Sinne behauptet Manfred Schneider (und das korrespondiert mit der Kälte-Diagnose von Baudrillard), heute würden wir

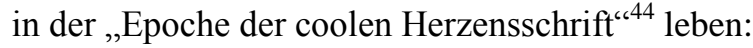

Das coole Zeichen des elektronischen Zeitalters ist der Klingelton des Mobiltelefons oder ein Signal des Computers, dass eine neue Mail eingetroffen ist. Das kann alle paar Minuten geschehen und so eine Nachricht oder eine Stimme des Geliebten ankündigen. ${ }^{45}$

Der Umstand, dass eine medienspezifische (schnelle und kalte) Kommunikation nicht auf das Begehren nach dem Anderen setzt, sondern auf einen infantilen Narzissmus hinausläuft, wird inzwischen zum Thema von Kunst und Literatur. In Elfriede Jelineks Winterreise (2011) kommt das ganze Vergnügen aus dem ,riesigen Netz“, das den Raum einnimmt, ,wo früher die Sonne war, wo früher der Mond war, diese riesige erdumspannende Möglichkeit auf Genuß, auf Spaß, auf ein Teilen von Freuden, auf ein Mitteilen von Leiden, auf ich-weiß-nicht-was ${ }^{\text {‘46 }}$. Diese Möglichkeit „klafft“ „wie eine riesige Vulva, denn alle wollen ja lustig sein“ ${ }^{47}$ Sämtliche Wünsche werden im Netz sofort befriedigt ${ }^{48}$, ohne dass man in diesen virtuellen Aben-

${ }^{43}$ Evelyn Schlag: Das L in Laura. Roman. Wien 2003, S. 211-212.

${ }^{44}$ Manfred Schneider: Herzensschriften. Liebesbriefe und Liebesroman. In: Peter Kemper, Ulrich Sonnenschein (Hrsg.): Liebe. Zwischen Sehnsucht und Simulation. Frankfurt a. M. 2005, S. 276-286, hier S. 276.

${ }^{45} \mathrm{Ebd}$

${ }^{46}$ Elfriede Jelinek: Winterreise. Reinbek bei Hamburg 2011, S. 60.

${ }^{47}$ Ebd.

${ }^{48} \mathrm{Zu}$ der Kälte- und Kommunikationsproblematik in Winterreise vgl. Ulrike Vedder: Kältelehren der ,Winterreise“. In: Colloquia Germanica, Bd. 43, Heft 1/2 (2013), Sonderband: Cold fronts. Kältewahrnehmungen in Literatur und Kultur vom 18. bis 20. Jahrhundert. Hrsg. von Inge Stephan und Monika Szczepaniak, S. 131-145. 
teuern auf den Anderen eingehen und Sicherheit verlieren oder gar ein Risiko des unkontrollierten Begehrens eingehen muss.

„Doch alle Lust will Ewigkeit“49 - sagt Nietzsches Zarathustra - und das Begehren steht im Zeichen der Negativität. Dem Begehren ist nämlich eine Mangelstruktur immanent: Es ist unstillbar, denn keine Liebe, keine Präsenz des Anderen, keine Eroberung schließlich - auch im Sinne von Aneignung, Unterordnung, Machtgewinn - kann vollständig genügen, stets bleibt ein Rest an Unbefriedigtsein zurück:

Das Begehren weiß nämlich gar nicht, was es will. Es handelt grundlos und lehnt jede Bemühung, sich durch Wiederholung bestätigt zu sehen, ab. Als unkontrollierte Kraft bricht es in etablierte Ordnungen ein, um dort innerhalb des lang schon etablierten, kodifizierten Sinns einen Gegen-Sinn zu schaffen $[\ldots] .{ }^{50}$

Die ambivalente und komplizierte Struktur des Begehrens, das immer einen Mangel, eine Negativität ${ }^{51}$ bedeutet, impliziert eine Spannung zwischen dem Verlangen nach einer Vereinigung mit dem Anderen und dem Verlangen nach Differenz. Darüber hinaus wohnt den Befriedigungsversuchen die Erfahrung inne, ,dass man über das eigene Begehren und dessen Erfüllung auch das Begehren des anderen begehrt und damit auch erfährt, daß der andere sich begehrt wünscht ${ }^{\text {‘52 }}$.

Das Begehren setzt immer die Erfahrung eines Abstands zwischen dem Aufkommen und der Befriedigung voraus, es bedeutet Warten und Sehnsucht. Warten und Sehnsucht werden mit Wärme, mit einer Sprache der Leidenschaft, mit Genuss oder dessen Erwartung assoziiert. Was wird aber aus dem Begehren, wenn das Warten auf ein Minimum schrumpft oder gar entfällt, wenn es keine Distanzen mehr gibt, wenn eine (mögliche) Anwesenheit durch permanente virtuelle Nähe ersetzt wird? Die Antwort von Manfred Schneider lautet: „Die erotische Kommunikation wird maschinenartig, cool und verrückt. ${ }^{{ }^{653}}$ Die schnelle Befriedigung der elektronischen Kommunikation nivelliert sowohl die Spannung der Begegnung in realen Räumen, die früher als Kontaktbörsen fungierten (Oper, Theater, Ball etc.), als auch die heiße Glut und Intensität des Begehrens in der Phase des Wartens, des SichSehnens nach der leiblichen Präsenz des/der Geliebten. ${ }^{54}$ Eine wirtschaftliche Philo-

${ }^{49}$ Friedrich Nietzsche: Also sprach Zarathustra. Kritische Studienausgabe Bd. 4. Hrsg. von Giorgio Colli und Mazzino Montinari. München 1999, S. 401-402.

${ }^{50}$ Umberto Galimberti: Die Sache mit der Liebe. Eine philosophische Gebrauchsanweisung. Aus dem Italienischen von Annette Kopetzki. München 2007, S. 85.

${ }^{51}$ Vgl. beispielsweise die phänomenologische Konzeption von Renaud Barbaras, der von einer „unbestimmbaren Negativität“ spricht. Mehr dazu: Gondek, Tengelyi: Neue Phänomenologie in Frankreich, Kap. 5, Zitat S. 595.

${ }^{52}$ Niklas Luhmann: Liebe als Passion. Zur Codierung von Intimität. Frankfurt a. M. 1994, S. 33.

${ }^{53}$ Schneider: Herzensschriften, S. 282.

${ }^{54}$ Freilich macht Aaron Ben-Ze'ev auf die paradoxe Natur der Online-Beziehungen aufmerksam und unterstreicht, diese hätten zwar einen virtuellen Charakters, die Emotionen, die sie hervorrufen, 
sophie der Liebe ${ }^{55}$ mit der ihr eingeschriebenen rationalen Kalkulation wechselseitiger Lust, der vernetzten Intimität, der computergesteuerten Paarbildung bzw. den auf Kompatibilität basierenden Partnerschaften trägt zur „Verwischung der Grenzen zwischen real/virtuell, vertraut/fremd, nah/' $a l s-o b-n a h{ }^{\prime \prime}{ }^{366}$ bei.

Auf die Auswirkung völlig neuer Formen der Kommunikation auf kulturelle Lebensstile sowie allgemein auf den Begriff von Realität und den Umgang damit verweist Günther A. Höfler im Aufsatz über die Prosa der Generation Y, einer Generation, welche aus den ersten Digital Natives bestehe, die online aufgewachsen seien. ${ }^{57}$ Das Krisengefühl - die immerwährende Unsicherheit, Überfordertheit und Sinnleere - färbt als eine prägende Erfahrung nicht zuletzt auf Beziehungsverhältnisse ab. „Manjas Generation sei so abgeklärt und kritisch, sie glaubten nicht mehr [...] an den Zauber zwischen Mann und Frau" - in diesem Zitat aus dem Erzählband Der Schädel von Madeleine. Paargeschichten (2014) von Lara Freundenthaler sieht Höfler ,die postromantische Haltung ${ }^{\text {“58 }}$ der Kohorte pointiert und hält fest, die authentischsten Erzählstimmen postromantischer Liebeserzählungen seien in der Mehrzahl „rückhaltlos, direkt, cool und trashig“. 59

Julia Kristeva schrieb einmal: „Die Liebe ist im Grunde ein Schmerz, ein Wort oder ein Brief. ${ }^{“ 60}$ Vor dem Hintergrund der wahrnehmbaren Abgeklärtheit scheint die Liebe dies nicht mehr zu sein, was in Verbindung mit der Abkühlung der heißen Herzensschriften bzw. dem medialen Klimawandel in Richtung Kälte/Coolness zum Erkalten oder gar zum Schwinden des Begehrens führt. Hier erfolgt eine semantische Verschiebung bzw. Re-Konzeptualisierung der Begriffe, die mit hohen Temperaturen verknüpft waren: In der romantischen Poesie ging das Begehren mit der Herzensglut einher, während der Winter für das Ende der Liebe stand, für das Erstarren der Gefühle, für enttäuschte Hoffnungen - man denke etwa an den Topos der

seien aber nicht nur durchaus real, sondern auch sehr intensiv - vgl. Aaron Ben-Ze'ev: Love Online. Cambridge 2004.

${ }^{55}$ Diese These liegt den Ausführungen von Eva Illouz zugrunde, die folgende soziologische Diagnose formuliert: „Wo es in der Produktionssphäre also darum ging, Emotionen ins Zentrum sozialer Beziehungen zu stellen, fixierten sich intime Beziehungen zunehmend auf politische und ökonomische Modelle des Handels und des Tausches.“ (Eva Illouz: Gefühle in Zeiten des Kapitalismus. Frankfurter Adorno-Vorlesungen 2004. Aus dem Englischen von Martin Hartmann. Frankfurt a. M. 2007, S. 60-61).

${ }^{56}$ Joanna Drynda: ,, Schreiben ist wie küssen, nur ohne Lippen. Schreiben ist küssen mit dem Kopf. “ Erotische Begegnungen im Cyberspace, in Gegenwartsromanen inszeniert. In: Doris Moser, Kalina Kupczyńska (Hrsg.): Die Lust im Text. Eros in Sprache und Literatur. Wien 2009, S. 399-412, hier S. 399.

${ }^{57}$ Günther A. Höfler: »Definitely Maybe« Zur Prosa der Generation Y. In: Text+Kritik. Zeitschrift für Literatur. Bd. IX (2015). Sonderband: Österreichische Gegenwartsliteratur. Hrsg. von Hermann Korte, S. 310-321, hier S. 311.

${ }^{58}$ Ebd., S. 317.

${ }^{59} \mathrm{Ebd}$

${ }^{60}$ Julia Kristeva: Geschichten von der Liebe. Aus dem Französischen von Dieter Hornig und Wolfram Bayer. Frankfurt a. M. 1989, S. 14. 
Herzenskälte. Noch bei Ingeborg Bachmann wird die Imagination der Liebe in Der gute Gott von Manhattan als „Flammenschrift" ${ }^{\text {“61 }}$ beschworen; im Gedicht Erklär mir, Liebe vermittelt die Feuermetaphorik die Intensität des erotischen Begehrens, das sich gegen den einseitigen, kalten Umgang mit Gedanken behauptet; ihre „flammenden Briefe“, „flammenden Aufrufe“, „flammenden Begehren“62 reflektiert die Ich-Erzählerin in einem Gespräch mit Malina. In einer ähnlichen Form erscheint ein poetisches Bild des Begehrens, das auf die Metaphorik der Hitze zurückgreift, derzeit in Nora Gomringers Gedicht Brandstifter aus dem Band Klimaforschung (2008): ,Du ein Brand in mir/ Mein Herz brandblasig [...].663

In Bezug auf die gegenwärtige Gefühlskultur konstatiert Wolfgang Emmerich einen ,pragmatisch-bastlerischen Umgang mit den, dem Anspruch nach, höchsten Gefühlen des Menschen “64 und er verweist auf das geschrumpfte Begehren in den großen Romanen des 20. Jahrhunderts. Eva Illouz diagnostiziert die Hegemonie einer entzauberten, rationalisierten, leidenschaftslosen Liebe, die wie eine „Religion ohne Glauben “65 funktioniert. Norbert Bolz analysiert das unstillbare Begehren in Verbindung mit dem Konsum und bezeichnet das Prinzip Sein ist Erregtsein als einen Grundsatz des Marketings in Sachen Liebe ${ }^{66}$ Und Rüdiger Safranski stellt in seinem Aufsatz mit dem vielsagenden Titel Der erkaltete $\operatorname{Eros}^{67}$ fest, dass das „heiBe Thema" der Erotik selbst im Forschungsfeld der Geistes- und Sozialwissenschaften nur noch „kalte Theorien“ hervorbringt - eine „unterkühlte“ Wissenschaft sei u. a. die Kultursoziologie der Liebe.

Bezeichnenderweise spürt man in der Gegenwartskultur gleichzeitig einen allgemeinen Drang nach Gefühl und Pathos, was Marie-Luise Angerer zufolge unterdessen als ein Dispositiv betrachtet werden könnte, ,in dem philosophische, kunstund medientheoretische Diskurse mit molekularbiologischen, kybernetischen und

${ }^{61}$ Ingeborg Bachmann: Werke 1: Gedichte, Hörspiele, Libretti, Übersetzungen. Hrsg. von Christine Koschel, Inge von Weidenbaum, Clemens Münster. München/Zürich 1993, S. 316.

${ }^{62}$ Ingeborg Bachmann: Werke 3: Todesarten: Malina und unvollendete Romane. Hrsg. von Christine Koschel, Inge von Weidenbaum, Clemens Münster. München/Zürich 1993, S. 245.

${ }^{63}$ Nora Gomringer: Klimaforschung. Leipzig 2008, S. 27.

${ }^{64}$ Wolfgang Emmerich: Liebe als Passion? Nein danke. Vom Schwinden des Begehrens im Spiegel der Popliteratur. In: Heinz-Peter Preusser, Anthonya Visser (Hrsg.): Alltag als Genre. Heidelberg 2009, S. 133-148, hier S. 134.

${ }^{65}$ Eva Illouz: Eine Religion ohne Glauben: Liebe und die Ambivalenz der Moderne. In: Yvonne Niekrenz, Dirk Villányi (Hrsg.): LiebesErklärungen. Intimbeziehungen aus soziologischer Perspektive. Wiesbaden 2008, S. 212-220.

${ }^{66}$ Norbert Bolz: Das Begehren und der Konsum. In: Peter Kemper, Ulrich Sonnenschein (Hrsg.): Das Abenteuer Liebe. Bestandsaufnahme eines unordentlichen Gefühls. Frankfurt a. M. 2004, S. 233-239.

${ }^{67}$ Rüdiger Safranski: Der erkaltete Eros. In: Moritz Baßler, Ewout van der Knaap (Hrsg.): Die (k)alte Sachlichkeit. Herkunft und Wirkungen eines Konzepts. Würzburg 2004, S. 9-17. Zu den Theorien im Kontext der „Krise des Begehrens“ vgl. Thomas Gebel: Krise des Begehrens: Theorien zur Sexualität und Geschlechterbeziehung im späten 20. Jahrhundert. Hamburg 2002. 
kognitionspsychologischen zu einer neuen ,Wahrheit des Menschen' verlötet werden“68. Diese „,affektive Epidemie“ (Jackie Stacey) scheint alle möglichen Lebensbereiche zu durchdringen, nur nicht die der Liebe und des Begehrens. Allerdings sei die Liebe, so der deutsche Sexualwissenschaftler Volkmar Sigusch, „allgemeines Erfordernis in der Kälte des Lebens“:

Die Leere, Distanz und Kälte der Arbeitswelt, überhaupt des gesellschaftlichen Lebens, sind im Allgemeinen nur mit der Nähe und Wärme einer Liebesbeziehung auszuhalten, die wenn schon nicht zu erreichen, so doch wenigstens versprochen sind. ${ }^{69}$

Gegen wissenschaftlich-kalte Erklärungsmuster wird Liebe neulich von der Philosophin Angelika Krebs leidenschaftlich verteidigt:

Wir leben in einer hektischen Zeit, in der das Haus der Liebe durch steigende Flexibilitätsanforde-
rungen und Erreichbarkeitserwartungen aus der ökonomischen Sphäre bedroht ist. Unsere Welt ist
vollgestellt mit bloßen simulacra von Intimität: flachen Facebookfreundschaften oder seelenlosem
Sex. Und dann hat auch noch ,die Wissenschaft festgestellt“, dass Liebe ohnehin nur eine Sache
der Hormone oder der Gene ist. - Das ist natürlich Unsinn. Liebe ist eines der wichtigsten Dinge im
menschlichen Leben. Man überlässt so etwas nicht den Hormonen oder den Genen. Und man gibt
sich auch nicht mit billigem Ersatz zufrieden. ${ }^{70}$

Könnte also auch nicht heute noch mit dem lyrischen Ich des Gedichts Brausen von Robert Walser behauptet werden: „Dann gibt es Stunden, wo ich weiß,/ daß wir vor Liebe alle hei $\beta^{\text {، } 71}$ ? Denn das Begehren evoziert immer eine Perspektive auf Befriedigung, auch wenn dies nur auf Konstruktionen von Trugbildern hinauslaufen mag.

Die zuvor formulierte These von der merklichen Klimaabkühlung in Sachen Liebe und Begehren lässt sich am Beispiel vieler Literatur- und Kunstwerke nachweisen. Es stellt sich aber die Frage, wie diese im Einzelnen auf facettenreiche Transformationen der Gefühlskultur reagieren? Begegnen sie den heutigen „reinen Beziehungen“ (Anthony Giddens) und „sachlichen Romanzen“ (Erich Kästner) mit einer jubilatorischen Geste oder artikulieren sie die alte romantische Figuration von Liebe, Körpernähe, Leidenschaft und Wärme? Oder haben sie ein überraschendes drittes Szenario parat, etwa die Dekonstruktion des hierarchisch organisierten Gegensatzpaares kalt warm? Jedenfalls: Goethes Werther wähnt sich in der Entfernung von einer halben Stunde ,zu nah in der Atmosphäre““72 der Geliebten, er spricht von der „warmen Freu$\mathrm{de}^{\text {“" an ihren Handlungen }}{ }^{73}$, vom heißen Verlangen nach ihr, und bei dem Gedanken,

${ }^{68}$ Marie-Luise Angerer: Vom Begehren nach dem Affekt. Zürich/Berlin 2007, S. 7.

${ }^{69}$ Volkmar Sigusch: Neosexualitäten. Über den kulturellen Wandel von Liebe und Perversion. Frankfurt a. M./New York 2005, S. 15.

${ }^{70}$ Krebs: Zwischen Ich und Du, S. 12 [Kursive im Original].

${ }^{71}$ Robert Walser: Brausen. In: Rainer Brambach (Hrsg.): Moderne deutsche Liebesgedichte. Von Stefan George bis zur Gegenwart. Zürich 1980, S. 24.

${ }^{72}$ Johann Wolfgang Goethe: Die Leiden des jungen Werther. Fassung von 1787. Leipzig 1981, S. 37.

${ }^{73}$ Ebd., S. 38. 
dass sie ihn wohl liebe, ,durchglüht“ ihn das Wonnegefühl. ${ }^{74}$ Die Beiträge des Bandes Temperaturen des Begehrens, der mit einem kulturwissenschaftlich-meteorologischen Zugang eine neue Perspektive auf das ewig aktuelle Thema zu bieten versucht, fokussieren die kulturellen Texte des 20. und 21. Jahrhunderts - einen emotionsgeschichtlichen Kontext, in dem sich ein Übergang vollzieht „,von einem Begehren, das sich aus dem Mangel speist, zu einem Begehren, das der Überfülle des Seins geschuldet ist“ ${ }^{75}$ Gefragt wird nach den Konstellationen von Temperatur und Begehren in Verbindung mit Liebe und Erotik, auch im Kontext der Intersexualität, mit Politik, Machtergreifung und gesellschaftlichem Umbruch, mit patriarchalen und kolonialen Herrschaftsstrukturen und mit medialen Darstellungskonventionen.

Die vorliegenden Aufsätze untersuchen Räume und Inszenierungsformen von Kälte und Wärme in den „Kulturlandschaften des Herzens“ (Volker Demuth) und in breiteren gesellschaftlichen Dimensionen. Sie fokussieren literarische bzw. ikonographische Einbrüche von verschiedenen Temperaturen, die analytische, ironische oder kritische Subtexte generieren und sich als Gegendiskurse bezeichnen lassen. Sie berücksichtigen auch intellektuelle und ökonomische Faktoren, die neue Varianten von Gefühlskultur oder „Intimitätsökonomie“ hervorbringen; gendering-Aspekte von Emotionen und Temperaturen und ihren Einfluss auf die Intensitäten des Begehrens; und nicht zuletzt den Zusammenhang von Medienräumen und Medienformaten mit den Temperaturen des Begehrens sowie die Spiegelung dieser Problematik in der sekundären Medienwirklichkeit der Literatur und Kunst. Teilweise überschneiden sich die Ansätze, teilweise gehen sie weit auseinander, dennoch ergibt sich eine gewisse Schwerpunktsetzung. Dagmar von Hoff konzentriert sich auf meteorologische Angaben sowie die Inszenierung einer scheinbar schön-warmen Temperatur in der Anfangspassage des Romans Der Mann ohne Eigenschaften und zeigt an diesem Beispiel Robert Musils Strategien, Historizität und Narrativität zu verschränken. In Anlehnung an die Temperaturen als ein aufwertendes bzw. abwertendes Prinzip der kolonialen Denkweise deutet Swati Acharya den Topos des kolonialen Begehrens in den Romanen Das Paradies des August Engelhardt von Marc Buhl, Imperium von Christian Kracht und Der Weltensammler von Ilija Trojanow. Ariane Martin richtet die Aufmerksamkeit auf das technische Instrument Thermometer und fragt systematisch nach dem Zusammenhang zwischen diesem ,kühlen' medizinischen Messgerät und dem metaphorisch vermittelten ,heißen' Begehren in Thomas Manns Roman Der Zauberberg. Die Versuche, die nicht empirisch messbare erotische Gefühlsintensität thermographisch zu fassen, sie also in Wärmegraden wie Hitze oder Kälte metaphorisch zu beschreiben, stehen auch im Mittelpunkt der weiteren Beiträge. Bożena Chotuj erläutert die thematische Zusammenführung von krankhaften, fiebe-

\footnotetext{
${ }^{74}$ Ebd., S. 108. Nur in dem Moment, als ihm bewusst wird, dass sein Dasein neben Lotte hoffnungslos ist, packt ihn „gräßliche Kälte“ (ebd., S. 97).

${ }^{75}$ Angerer: Vom Begehren nach dem Affekt, S. 8.
} 
rigen Zuständen und Geschlechterbeziehungen im Werk von Stanislaw Przybyszewski und zeigt die Ambivalenz des Begehrens, die die Figuren erfahren und erleben, indem sie zwischen Normerwartung und -erfüllung taumeln. Monika Szczepaniak nimmt die Figur des maßlosen Erotikers Don Juan in den Blick und analysiert Don Juan-Figuren von Ödön von Horváth und Robert Menasse als literarische Produkte von Gefühlskulturen, für die eine jeweils spezifische Affirmation der Kälte charakteristisch ist. Die Beobachtung, dass die Kälte sowohl in emotionaler als auch in meteorologischer Hinsicht in Romanen von Antje Rávic Strubel und Uwe Timm dominiert, ist richtungsweisend für Inge Stephans Untersuchung des inszenierten Beziehungsklimas. Während Inge Stephan die Trostlosigkeit der hetero- und homosexuellen (lesbischen) Beziehungen thematisiert, stellt Marta Wimmer am Beispiel des Romans Salamander von Jürgen Lodemann das non-konforme Begehren einer intersexuellen, in ein Liebesdreieck verwickelten Figur dar und befasst sich mit der anatomischen Besonderheit, die der Protagonistin einen fließenden Übergang im Bereich des Sexuellen ermöglicht und sich zugleich als „,doppelt lustvoll“ erweist. Den Weg von der „Wollust in die Wohllust“ (Volkmar Sigusch) verfolgt im Romanwerk von Stephan Thome Joanna Drynda und weist nach, in welchem Maße eine feste Beziehung, in der sich die Partner aufgehoben fühlen, in der empfundenen gesellschaftlichen Kälte als ein Refugium imaginiert wird. Auf die Wärme- und Kältemetaphorik wird auch dann zurückgegriffen, wenn transmediale Bezüge zur Debatte stehen. Janin Aadam setzt sich mit medialen Umsetzungen von Goethes Ballade Erlkönig auseinander und fokussiert dabei die Inszenierung des Erlkönigs als eines kalten Verführers im Gedicht selbst und in der filmischen Adaption Brennendes Geheimnis. Alexandra Tacke geht dem Problem der Darstellbarkeit von der weiblichen Stimme des Begehrens in Arthur Schnitzlers Novelle Fräulein Else nach und verweist auf visuelle Neuintepretationen des Werkes, von denen Manuele Fiors Comic Fräulein Else auf Kolorierung der Emotionen hin gründlich untersucht wird. Der letzte Beitrag von Rafal Pokrywka nimmt die neuesten Liebesromane, die nach Fifty Shades of Gray erschienen sind, in den Fokus, analysiert die stilistische Ebene der Texte (vor allem die Temperaturmetapher) und polemisiert mit dem Essay Die neue Liebesordnung von Eva Illouz. Die Hard-Core-Liebesromane erweisen sich als konventionelle Kulturartefakte mit geringer rhetorischer Kraft, demzufolge auch als wenig geeignet, große gesellschaftliche Konzepte zu tragen.

\section{Literatur}

Angerer, Marie-Luise: Vom Begehren nach dem Affekt. Zürich/Berlin 2007.

Bachmann, Ingeborg: Werke 1: Gedichte, Hörspiele, Libretti, Übersetzungen. Hrsg. von Christine Koschel, Inge von Weidenbaum, Clemens Münster. München/Zürich 1993.

Bachmann, Ingeborg: Werke 3: Todesarten: Malina und unvollendete Romane. Hrsg. von Christine Koschel, Inge von Weidenbaum, Clemens Münster. München/Zürich 1993. 
Ben-Ze'ev, Aaron: Love Online. Cambridge 2004.

Böhme, Gernot: Das Wetter und die Gefühle. Für eine Phänomenologie des Wetters. In: Kerstin Andermann, Undine Eberlein (Hrsg.): Gefühle als Atmosphären. Neue Phänomenologie und philosophische Emotionstheorie. Berlin 2011, S. 153-166.

Bolz, Norbert: Das Begehren und der Konsum. In: Peter Kemper, Ulrich Sonnenschein (Hrsg.): Das Abenteuer Liebe. Bestandsaufnahme eines unordentlichen Gefühls. Frankfurt a. M. 2004, S. 233-239.

Demmerling, Christoph: Gefühle, Sprache und Intersubjektivität. Überlegungen zum Atmosphärenbegriff der Neuen Phänomenologie. In: Andermann, Eberlein: Gefühle als Atmosphären, S. 43-55.

Demuth, Volker: Unnahbare Nähe. Von Liebe und der neuen Intimitätsökonomie. In: allmende. Zeitschrift für Literatur 86 (2010), S. 39-47.

Drynda, Joanna: ,, Schreiben ist wie küssen, nur ohne Lippen. Schreiben ist küssen mit dem Kopf. “Erotische Begegnungen im Cyberspace, in Gegenwartsromanen inszeniert. In: Doris Moser, Kalina Kupczyńska (Hrsg.): Die Lust im Text. Eros in Sprache und Literatur. Wien 2009, S. 399-412.

Emmerich, Wolfgang: Liebe als Passion? Nein danke. Vom Schwinden des Begehrens im Spiegel der Popliteratur. In: Heinz-Peter Preusser, Anthonya Visser (Hrsg.): Alltag als Genre. Heidelberg 2009, S. 133-148.

Galimberti, Umberto: Die Sache mit der Liebe. Eine philosophische Gebrauchsanweisung. Aus dem Italienischen von Annette Kopetzki. München 2007.

Gebel, Thomas: Krise des Begehrens: Theorien zur Sexualität und Geschlechterbeziehung im späten 20. Jahrhundert. Hamburg 2002.

Goethe, Johann Wolfgang: Die Leiden des jungen Werther. Fassung von 1787. Leipzig 1981.

Goethe, Johann Wolfgang: Versuch einer Witterungslehre. In: Goethes Werke: Vollständige Ausgabe letzter Hand. Bd. 51. Stuttgart/Tübingen 1834, S. 247-282.

Gomringer, Nora: Klimaforschung. Leipzig 2008.

Gondek Hans-Dieter, László Tengelyi (Hrsg.): Neue Phänomenologie in Frankreich. Berlin 2011.

Haselstein, Ulla: Coolness. In: Gunter Gebauer, Markus Edler (Hrsg.): Sprachen der Emotionen. Kultur, Kunst, Gesellschaft. Frankfurt a. M./New York 2014, S. 211-225.

Hermann, Judith: Sommerhaus, später. Erzählungen. Frankfurt a. M. 2009.

Höfler, Günther A.: »Definitely Maybe« Zur Prosa der Generation Y. In: Text+Kritik. Zeitschrift für Literatur. Bd. IX (2015). Sonderband: Österreichische Gegenwartsliteratur. Hrsg. von Hermann Korte, S. 310-321.

Homscheid, Thomas: Sexualzynismen in der Literatur des 20. Jahrhunderts. Am Beispiel von Erich Kästners „Fabian“ (1931). In: Stefan Neuhaus (Hrsg.): Figurationen der Liebe in Geschichte und Gegenwart, Kultur und Gesellschaft. Würzburg 2012, S. 257-272.

Hutfless, Esther: In Dir mehr als Dich. Phänomenologien des Begehrens zwischen diskursiver Produktion und leiblichem Zur-Welt-Sein. Würzburg 2011.

Illouz, Eva: Eine Religion ohne Glauben: Liebe und die Ambivalenz der Moderne. In: Yvonne Niekrenz, Dirk Villányi (Hrsg.): LiebesErklärungen. Intimbeziehungen aus soziologischer Perspektive. Wiesbaden 2008, S. 212-220.

Illouz, Eva: Gefühle in Zeiten des Kapitalismus. Frankfurter Adorno-Vorlesungen 2004. Aus dem Englischen von Martin Hartmann. Frankfurt a. M. 2007.

Krebs, Angelika: Zwischen Ich und Du. Eine dialogische Philosophie der Liebe. Berlin 2015.

Kristeva, Julia: Geschichten von der Liebe. Aus dem Französischen von Dieter Hornig und Wolfram Bayer. Frankfurt a. M. 1989.

Lentz, Michael: Liebeserklärung. Roman. Frankfurt a. M. 2003.

Luhmann, Niklas: Liebe als Passion. Zur Codierung von Intimität. Frankfurt a. M. 1994.

Meyer-Sickendiek, Burkhard: Gefühlstiefen: Aktuelle Perspektiven einer vergessenen Dimension der Emotionsforschung. In: Gertrud Lehnert (Hrsg.): Raum und Gefühl. Der Spatial Turn und die neue Emotionsforschung. Bielefeld 2011, S. 26-48. 
Morgenstern, Christian: Der Seufzer. In: Moderne deutsche Liebesgedichte. Von Stefan George bis zur Gegenwart. Hrsg. von Rainer Brambach. Zürich 1980.

Nietzsche, Friedrich: Also sprach Zarathustra. Kritische Studienausgabe Bd. 4. Hrsg. von Giorgio Colli und Mazzino Montinari. München 1999, S. 401-402.

Nussbaum, Martha C.: Konstruktion der Liebe, des Begehrens und der Fürsorge. Drei philosophische Aufsätze. Übers. Joachim Schulte. Stuttgart 2002.

Ortega y Gasset, José: Über die Liebe. Meditationen. Deutsch von Helene Weyl. Stuttgart 1957.

Osswald-Rinner, Iris: Oversexed und underfucked. Über die gesellschaftliche Konstruktion der Lust. Wiesbaden 2011.

Rothmann, Ralf: Feuer brennt nicht. Roman. Frankfurt a. M. 2009.

Safranski, Rüdiger: Der erkaltete Eros. In: Moritz Baßler, Ewout van der Knaap (Hrsg.): Die (k)alte Sachlichkeit. Herkunft und Wirkungen eines Konzepts. Würzburg 2004, S. 9-17.

Scherke, Katharina: Auflösung der Dichotomie von Rationalität und Emotionalität? Wissenschaftssoziologische Anmerkungen. In: Sabine Flick, Annabelle Hornung (Hrsg.): Emotionen in Geschlechterverhältnissen. Affektregulierung und Gefühlsinszenierung im historischen Wandel. Bielefeld 2009, S. 23-42.

Schlag, Evelyn: Das L in Laura. Roman. Wien 2003.

Schmitz, Hermann: Der Gefühlsraum. System der Philosophie III/2. Bonn 1969.

Schmitz, Hermann: Gefühle als Atmosphären und das affektive Betroffensein von innen. In: Hinrich Fink-Eitel, Georg Lohmann (Hrsg.): Zur Philosophie der Gefühle. Frankfurt a. M. 1993, S. 33-56.

Schneider, Manfred: Herzensschriften. Liebesbriefe und Liebesroman. In: Peter Kemper, Ulrich Sonnenschein (Hrsg.): Liebe. Zwischen Sehnsucht und Simulation. Frankfurt a. M. 2005, S. 276-286.

Scruton, Roger: Sexual desire. A moral philosophy of the erotic. New York 1986.

Sigusch, Volkmar: Neosexualitäten. Über den kulturellen Wandel von Liebe und Perversion. Frankfurt a. M./New York 2005.

Stamm, Peter: Agnes. Roman. Frankfurt a. M. 2015.

Ussel, Jos van: Sexualunterdrückung. Geschichte der Sexualfeindschaft. Gießen 1977.

Vedder, Ulrike: Kältelehren der „Winterreise“. In: Colloquia Germanica, Bd. 43, Heft 1/2 (2013), Sonderband: Cold fronts. Kältewahrnehmungen in Literatur und Kultur vom 18. bis 20. Jahrhundert. Hrsg. von Inge Stephan und Monika Szczepaniak, S. 131-145.

Vendrell Ferran, Íngrid: Metaphern der Liebe. Alexander Pfänder und Hermann Schmitz. In: Andermann, Eberlein: Gefühle als Atmosphären, S. 245-264.

Walser, Robert: Brausen. In: Rainer Brambach (Hrsg.): Moderne deutsche Liebesgedichte. Von Stefan George bis zur Gegenwart. Zürich 1980.

Widmer, Peter: Subversion des Begehens. Eine Einführung in Jacques Lacans Werk. Berlin 1997. 